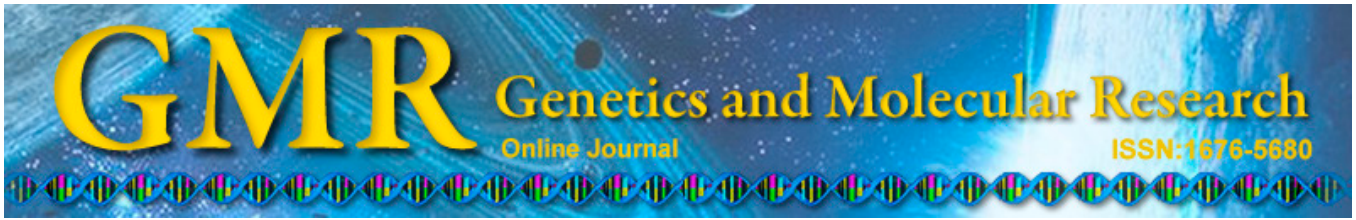

\title{
Effect of temperature on endogenous hormone levels and opposite phyllotaxy in maize leaf primordial
}

H. Ye, G.M. Han, Q. Ma, Y.Q. Tan, H.Y. Jiang, S.W. Zhu and B.J. Cheng

School of Life Sciences, Anhui Agricultural University, Hefei, China

Corresponding author: B.J. Cheng

E-mail: beijiucheng@ahau.edu.cn

Genet. Mol. Res. 14 (4): 17019-17027 (2015)

Received January 24, 2015

Accepted June 8, 2015

Published December 16, 2015

DOI http://dx.doi.org/10.4238/2015.December.16.2

\begin{abstract}
Newly identified maize (Zea mays) mutants with opposite leaf phyllotaxy are important in the study of the maize crop. Previous studies have revealed the developmental mechanism of opposite phyllotaxy on the physiological, cellular, and molecular levels. However, there have been few reports regarding the effects of changes in endogenous hormone levels in maize leaf primordia under different conditions. We conducted field studies to examine the influence of different environmental factors on leaf primordia differentiation. Our results indicated that compared with other major environmental factors, temperature was significantly positively correlated with the ratio of maize plants with opposite phyllotaxy. We examined endogenous hormone levels in maize at different temperatures using an enzymelinked immunosorbent assay. The results showed that the ratio of maize plants with opposite phyllotaxy was mainly influenced by the cytokinin/ auxin ratio. In addition, at the same temperature, the ratio of cytokinin/ auxin in maize with opposite phyllotaxy was significantly higher than that near isogenic lines with alternate phyllotaxy.
\end{abstract}

Key words: Cytokinin/auxin; Endogenous hormones; Temperature; Opposite phyllotaxy ratio; Phyllotaxy; Zea mays 


\section{INTRODUCTION}

High-yielding maize cultivars are very important crops, particularly cultivars with opposite leaf phyllotaxy, a highly valuable genetic resource causing traits such as a larger number female spikes and larger leaf areas, among other traits (Tian et al., 1994; Wang and Gu, 1995; Cheng, 2003). However, the mechanism underlying opposite phyllotaxy during leaf development remains unclear (Huang, 2003). Galinat (1971) identified a double phyllotaxis mutant and subsequently suggested that the trait is controlled by a single dominant gene (Galinat, 1972). Liu (1982) also identified a maize mutant with opposite phyllotaxy and suggested that this trait is unstable, with complex heredity, and that it evolved from the implicit phyllotaxy trait. However, these mutant lines exhibit several drawbacks such as dwarfism, genetic instability, an increased ratio of abnormal plants. Cheng et al. obtained a maize mutant that developed into a vertically opposite plant in 1990 (see Kan et al., 2003).

The opposite leaf trait is controlled by the transposition of the ZMRR3 gene, a dominant gene encoding a maize cytokinin (CTK) (Giulini et al., 2004), but this result differed from the results obtained by detecting and analyzing gene expression in different growth tips and stages using reverse transcription-polymerase chain reaction (Ma et al., 2008). Xie et al. (2002) identified 2 putative markers that are closely linked to 2 dominant genes for the opposite phyllotaxy trait in a maize opposite leaf and fruit mutant using random amplified polymorphic DNA analysis, and these markers were successfully transformed into sequence characterized amplified region markers. The two dominant genes were localized to specific simple sequence repeat markers of the second chain group (Tan et al., 2011). These results are very important for investigating the mechanism underlying the formation of the opposite phyllotaxy trait, despite the fact that the dominant genes for this train have not been identified. Previous studies indicated that indole-3-acetic acid (IAA) and CTK are the main hormones controlling leaf development (Skoog and Miller, 1957; Reinhardt et al., 2000; Ma et al., 2010). Using tissue culture experiments, Skoog and Miller (1957) found that IAA, CTK, and the balance between these hormones play key roles in leaf primordium differentiation. Ma (2010) showed that applying naphthaleneacetic acid to the shoot apical meristem changes the phyllotaxy in maize from opposite to alternate, and returns the plants to the original CTK/auxin ratio through exogenous hormone application of tissue culture-grown immature maize can cause the leaf primordia to return to the original phyllotaxis patterns.

Maize growth is controlled by several factors, such as endogenous hormone levels, nutrition, and the environment, among others (Zhao, 2009). To examine the effects of different temperatures on changes in endogenous hormone levels in maize leaf primordia, we examined the correlation between different endogenous hormone contents at different temperatures and the ratio between plants with the opposite trait in maize using enzyme-linked immunosorbent assay analysis.

\section{MATERIAL AND METHODS}

\section{Materials}

Seedling materials included the maize inbred lines 8701D, H4D, Dan3D, LiuD, ChangD, and WuMD. Among these, the opposite phyllotaxy traits of 8701D and H4D are stable, but those of the others are unstable. Liuh and $\mathrm{LiuDh}$ are the parents of the alternate 
phyllotaxy near isogenic lines of LiuD.

\section{Study location}

Field studies were conducted from 2000 to 2007 in areas of Hefei and Sanya, China, which are located at latitude $31^{\circ} 30^{\prime}$ and $32^{\circ} 37^{\prime}$ North and longitude $116^{\circ} 41^{\prime}$ and $117^{\circ} 52^{\prime}$ East, and $18^{\circ} 09^{\prime}$ and $18^{\circ} 37^{\prime}$ North and longitude $108^{\circ} 56^{\prime}$ and $109^{\circ} 48^{\prime}$ East, respectively.

\section{Site preparation}

Farmland with medium fertility was manually cleared. Using a randomized complete block design with 3 replicates, the land was divided into 3 blocks, each containing 8 plots that were $5.0 \times 5.0 \mathrm{~m}$. The distance between plants and lines was $0.4 \times 0.5 \mathrm{~m}$. The ratio of plants with opposite phyllotaxy was assessed at the 4-leaf seedling stage, and the results were confirmed again in 20- and 25-day-old seedlings.

\section{Treatments and experimental design}

Indoor experiments in maize were performed in artificial climate boxes. The conditions were controlled, with a relative humidity of $75 \%$, illumination of 10,000 $\mathrm{IX}$, and temperature gradients of $17^{\circ}, 21^{\circ}$, and $25^{\circ} \mathrm{C}$, which were the mean temperatures in the 3 different fields, respectively. The temperature was $2^{\circ} \mathrm{C}$ lower at night than in the daytime. Shoot apical meristems were separated from the stem apexes of young seedlings that were $3,6,9,12$, and 15 days old, with several seedlings examined at each time point.

The reaction kits for measuring endogenous hormone contents were purchased from the Plant Hormones Research Laboratory of the Agricultural University of China (Beijing, China) and the standard samples of plant hormones were purchased from Sigma (St. Louis, MO, USA). The scanning electron microscope and anatomical lenses S-3400N and SZ61 were purchased from Olympus Co. and Hitachi High-Tech. Co., respectively (Tokyo, Japan).

\section{Methods}

\section{Morphologic observation of leaf primordia}

Fifty plants were chosen randomly from 3 different groups from the 8701D, LiuD, and WuMD lines. The shoot apical meristems were observed and identified by SEM; the stem apex was obtained, fixed overnight under a vacuum in fluorescein diacetate, and dehydrated stepwise in $50,70,80,90$, and $100 \%$ alcohol. The ratios of samples with opposite phyllotaxy were analyzed statistically, and the contents of IAA and CTK were detected.

\section{Extraction of endogenous maize hormones}

To perform the enzyme-linked immunosorbent assay (Li and Zhou, 1996), the endogenous maize hormones were first extracted. First, $2 \mathrm{~mL}$ extraction solution $(80 \%$ alcohol containing 1-butylated hydroxytoluene) was combined with $0.5 \mathrm{~g}$ material derived from the growing tips of plants, which were ground into slurry in an ice bath under low light conditions. 
The mixture was incubated for $4 \mathrm{~h}$ at $4^{\circ} \mathrm{C}$ and centrifuged at $1000 \mathrm{~g}$ for $15 \mathrm{~min}$ at $4^{\circ} \mathrm{C}$. The precipitate was combined with $1 \mathrm{~mL}$ extraction solution. The liquid supernatant was filtered through a $\mathrm{C} 18$ column, dried under nitrogen in a water bath at $45^{\circ} \mathrm{C}$, and maintained at a constant volume by diluting $0.75 \mathrm{~mL}$ sample, which was prepared by weighing $8.0 \mathrm{~g} \mathrm{NaCl}, 0.2 \mathrm{~g}$ $\mathrm{NaH}_{2} \mathrm{PO}_{4}$, and $0.96 \mathrm{~g} \mathrm{Na}_{2} \mathrm{HPO}_{4} \cdot 12 \mathrm{H}_{2} \mathrm{O}$ and maintained at a constant volume by adding 100 $\mu \mathrm{L}$ Tween-20 to a final volume of $1000 \mathrm{~mL}$ with double-distilled water at $\mathrm{pH}$ 7.5. Each experiment was repeated 3 times to determine IAA content and endogenous cytokines, including zeatin riboside group, dehydro-zeatin riboside group, and isopentenyladenosine group, which together comprise total CTK content.

\section{Sample detection}

Using a previously described method ( $\mathrm{Li}$ and Meng, 1997), detection steps included coating, washing, competing, adding 2 antibodies, and adding substrate to reveal the color. Colorimetric assays were performed at $490 \mathrm{~nm}$, and the results were fitted to a logistic curve.

\section{RESULTS}

\section{Comparison of the ratios of maize inbred lines with opposite phyllotaxy grown in different areas and years}

Using various maize inbred lines with opposite phyllotaxy such as H4D, 8701D, Dan3D, LiuD, ChangD, and WuMD, we investigated seedlings grown in different areas and years. The results showed that the ratio of plants in the inbred lines with opposite phyllotaxy increased with increasing breeding age. The ratios for H4D and 8701D were maintained at high, stable levels in different areas, while for others, the ratios varied. Additionally, ratios between plants with opposite phyllotaxy were stable in Sanya and unstable in Hefei, as shown in Figure 1.

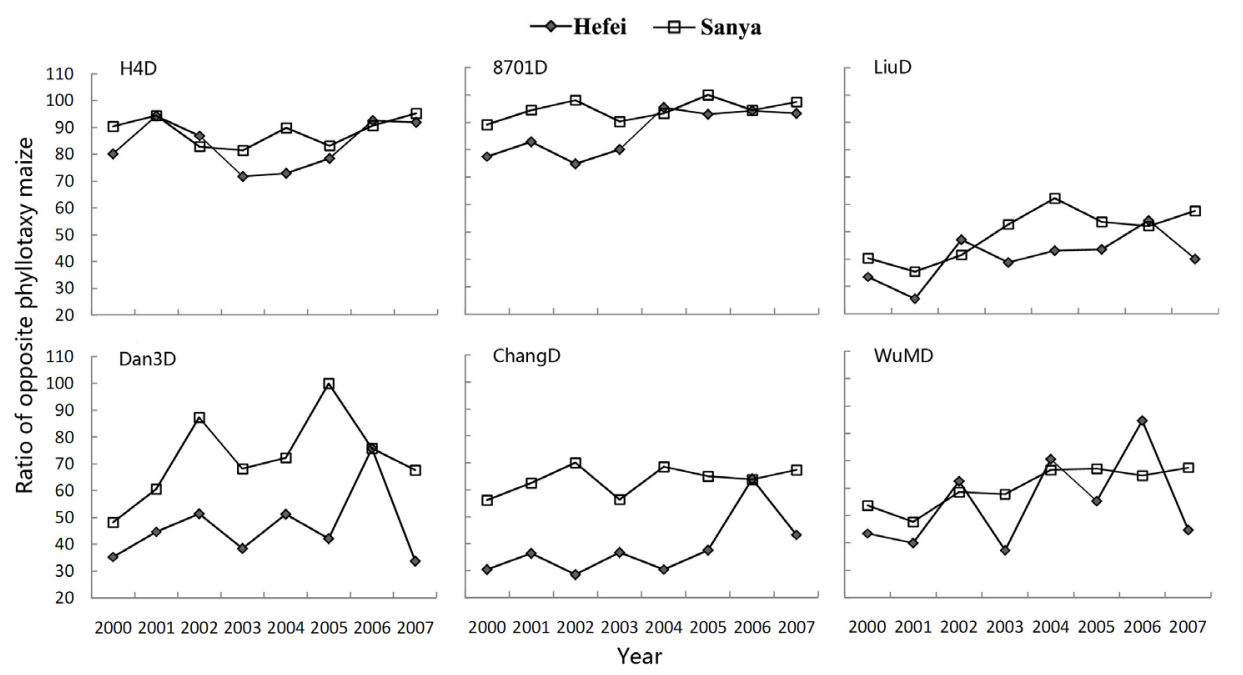

Figure 1. Differences in opposite phyllotaxy ratios in maize inbred lines in different areas and years. 
Crop growth is affected by several factors, particularly genotype and environmental conditions, which represent key internal and external factors, respectively. The results indicate that the inbred lines H4D and 8701D were mainly controlled by genotype, while the others were influenced by both factors. To clarify the effect of major climate conditions on the ratio of maize plants with opposite phyllotaxy, we performed correlation analysis, as shown in Table 1.

Compared with other environmental factors, temperature showed a significant positive correlation with the ratio of maize plants with opposite phyllotaxy, as shown in Table 1. For the inbred lines Dan3D, LiuD, ChangD, and WuMD, the ratios of plants with opposite phyllotaxy were higher and more stable in Sanya than in Hefei, possibly because the temperature was higher in Sanya at the seedling stage. In years in which temperatures were higher, including 2002, 2004, and 2006, the ratios of plants with opposite phyllotaxy were higher in Hefei (Figure 1).

Table 1. Correlation analysis between the major climate conditions and the opposite phyllotaxy ratios of different maize inbred lines.

\begin{tabular}{lcccccc}
\hline & \multicolumn{7}{c}{ Inbred lines } \\
\cline { 2 - 6 } & H4D & 8701D & Dan3D & liuD & ChangD & wuMD \\
\hline Environment factors & & & & & \\
Day mean temperature $\left({ }^{\circ} \mathrm{C}\right)$ & 0.363 & $0.623^{* *}$ & $0.836^{* *}$ & $0.658^{* *}$ & $0.900^{* *}$ & $0.577^{*}$ \\
Light time (h/day) & -0.025 & $0.570^{*}$ & 0.312 & 0.065 & 0.441 & -0.065 \\
Rainfall (mm/day) & 0.066 & -0.077 & -0.373 & -0.092 & -0.244 & 0.027 \\
\hline
\end{tabular}

$*, * *$ Significant difference at the 0.05 and 0.01 levels, respectively.

\section{Statistics of opposite phyllotaxy ratios}

By investigating the morphology of maize shoot apical meristems using scanning electron microscopy, we determined that the shoot apical meristems of maize with opposite phyllotaxy were larger than those of plants with alternate phyllotaxy, and the arrangement of shoot apical meristems differed between plants with opposite and alternate phyllotaxy. The ratios of plants with opposite phyllotaxy differed among inbred lines; the order of lines (from high to low opposite phyllotaxy ratios) was 8701D, LiuD, and WuMD, as shown in Figure 2. The opposite phyllotaxy ratios for LiuD and WuMD, but not $8701 \mathrm{D}$, were greatly influenced by temperature and were positively correlated with temperature. The order of lines, arranged by standard deviation (from high to low), was 8701D, LiuD, and WuMD, with values of 3.28, 10.35, and 14.22\%, respectively.

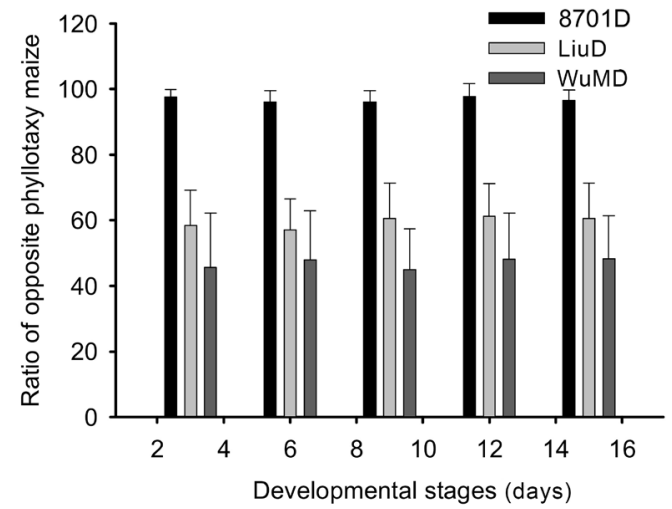

Figure 2. Opposite phyllotaxy ratios of different maize inbred lines at different temperatures. 


\section{Changes in the CTK/IAA ratios of maize inbred lines at different temperatures and growth stages}

The CTK/IAA ratios differed among inbred lines, and the changes generally corresponded to changes in temperature. While the CTK/IAA ratios in $8701 \mathrm{D}$ were similar at different temperatures, the other lines exhibited positive correlations with temperature. However, in some inbred lines, such as WuMD, the CTK/IAA ratio was higher at $17^{\circ} \mathrm{C}$ than at other temperatures (Figure 3).
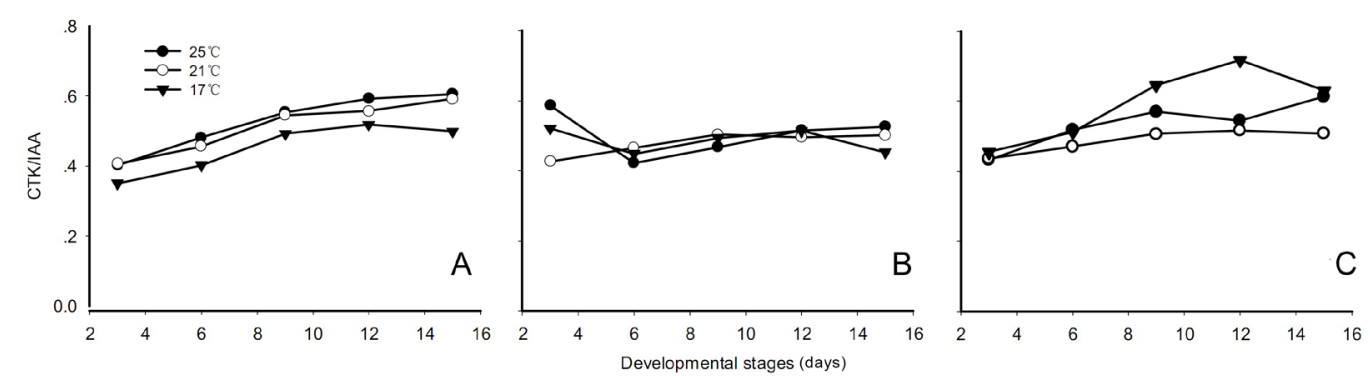

Figure 3. Differences in CTK/IAA ratios of maize inbred lines at different temperatures and developmental stages. A. LiuD, B. $8701 \mathrm{D}$, C. WuMD.

To examine the relationship between the CTK/IAA ratio and the opposite phyllotaxy ratio, we performed correlation analysis using the SPSS software (SPSS, Inc., Chicago, IL, USA). We observed a positive correlation between the CTK/IAA ratio and the ratio of plants with opposite phyllotaxy (except for the inbred line WuMD at $17^{\circ} \mathrm{C}$ ), with correlation coefficients of 0.6801 and 0.7178 for inbred lines 8701D and WuMD, respectively (Figure 4). In addition, for WuMD, the CTK/IAA ratios at both $21^{\circ}$ and $25^{\circ} \mathrm{C}$ exhibited positive correlations with the opposite phyllotaxy ratio, with correlation coefficients of 0.6930 and 0.8174 , respectively (Figure 5).
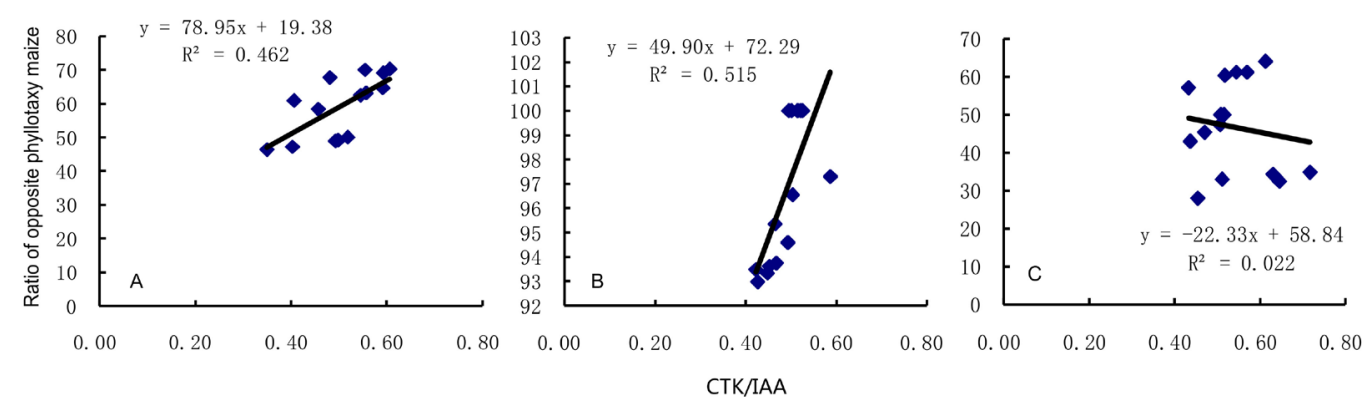

Figure 4. Analysis of the linear correlation between the ratios of opposite phyllotaxy maize and temperature. A. 8701D, B. LiuD, C. WuMD.

To examine the influence of temperature on the CTK/IAA ratios of 3 inbred lines, the data were analyzed using the SigmaPlot software (SYSTAT, San Jose, CA, USA). The results indicated that the CTK/IAA ratios of different inbred lines with opposite phyllotaxy differed; the order of lines, with ratios arranged from high to low, was 8701D, LiuD, and WuMD, with standard deviations of $0.03,0.04$, and 0.06 , respectively (Figure 6 ). The results also show that 
the changes in CTK/IAA ratios of different inbred lines were consistent with those of opposite phyllotaxy at different temperatures (Figure 2).
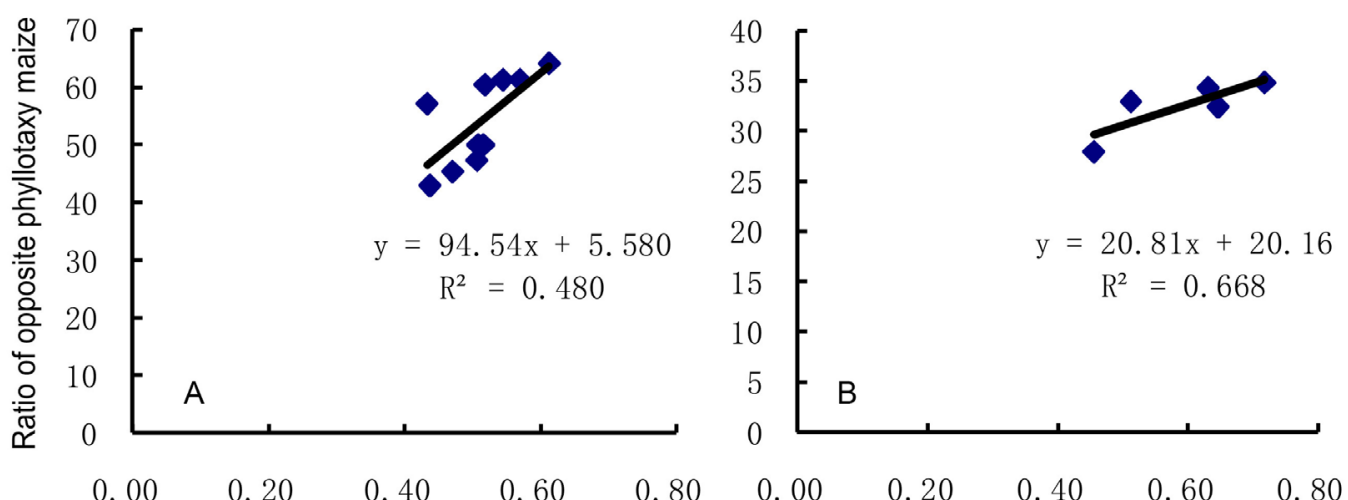
0. 00
0. 20
0.40
0.60
0.80
0.00
0.20
0.40
0.60
0.80
CTK/IAA

Figure 5. Linear correlation analysis on WuMD between the ratio of opposite phyllotaxy and temperature. A. $21^{\circ} \mathrm{C}$ and $25^{\circ} \mathrm{C}, \mathbf{B} .17^{\circ} \mathrm{C}$.

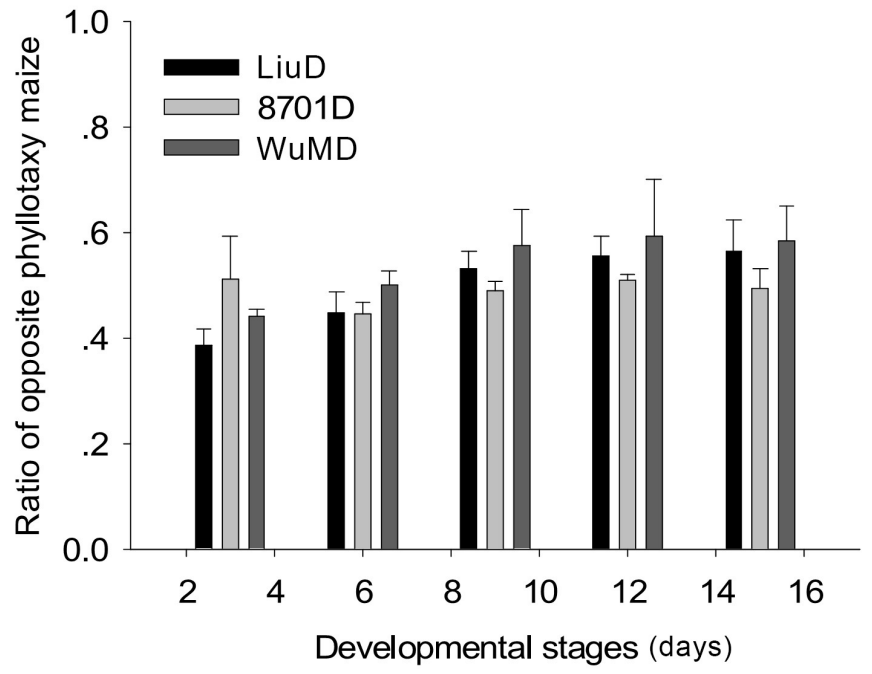

Figure 6. Dissociation of CTK/IAA of maize inbred lines at different temperatures.

\section{Changes in CTK/IAA ratios of different maize inbred lines at the same temperature}

We also analyzed LiuD, LiuDh, and Liuh, as shown in Figure 7. At the same temperature, the CTK/IAA ratio of LiuD was higher than that of LiuDh and Liuh. Among the alternate phyllotaxy maize inbred lines, the CTK/IAA ratio of LiuDh was always higher than that of Liuh and even higher than that of the opposite phyllotaxy maize inbred line LiuD grown at the lower temperature, indicating that the gene(s) controlling the opposite phyllotaxy character retain(s) a high expression index in alternate phyllotaxy maize inbred lines. 


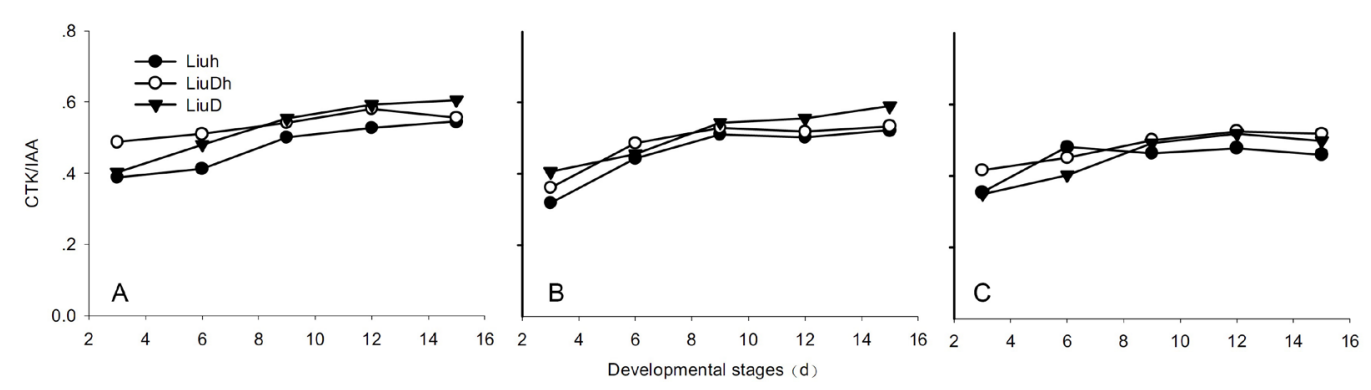

Figure 7. Change in the CTK/IAA content of different maize lines at the same temperature. A. $25^{\circ} \mathrm{C}, \mathbf{B} .21^{\circ} \mathrm{C}, \mathbf{C} .17^{\circ} \mathrm{C}$.

The experiments confirmed that the formation of the opposite phyllotaxy character was correlated with the CTK/IAA ratio at the growing point of maize stem tips, which played an important role in phyllotaxy differentiation. The balance of CTK to IAA is a key factor controlling the types of leaf primordia formed. Among the main environmental factors affecting the CTK/IAA ratio, including light exposure time, temperature, and rainfall, temperature showed the strongest effect on opposite phyllotaxy ratios of maize inbred lines.

\section{DISCUSSION}

Plant growth is controlled by multiple hormones, and particularly by the hormonal balance, as reported previously (Wang et al., 2008). Our results revealed negative correlations between the contents of gibberellin (GA), abscisic acid (ABA), and IAA and opposite phyllotaxy ratios in maize, but a positive correlation was observed for CTK. The results in our study also showed that the opposite phyllotaxy ratios in maize were mainly influenced by the ratio of CTK/IAA, but not by (GA + IAA + CTK)/ABA, CTK/ABA, or CTK/GA (data not shown).

Changes in temperature changed the CTK/IAA ratio and caused the opposite phyllotaxy ratio to fluctuate. However, in different maize inbred lines, the effects of temperature differed. The CTK/IAA ratios for LiuD and WuMD, but not 8701D, were strongly influenced by temperature. The CTK/IAA ratio may have a threshold value that is different for various inbred lines, leading to a positive correlation with the opposite phyllotaxy ratio within a certain range. Moreover, the current study indicated that the 9th day represented a turning point, after which the hormonal content in different maize lines was maintained within a relatively stable range.

At the same temperature, the CTK/IAA ratio in maize with opposite phyllotaxy was significantly higher than those of its near isogenic lines of alternate phyllotaxy maize, as was previously reported (Ma et al., 2008). The CTK/IAA ratio of the alternate phyllotaxy near isogenic lines of opposite phyllotaxy maize was always higher than that of the alternate phyllotaxy parent line, and both trends were consistent. These results indicate that the gene controlling the opposite phyllotaxy trait maintained a higher expression index in the near isogenic lines of opposite phyllotaxy maize, and the opposite phyllotaxy trait did not appear to be latent (Tian et al., 1994; Wang and Gu, 1995; Liu et al., 1998).

\section{ACKNOWLEDGMENTS}

Research supported by grants from the National Natural Science Foundation of China (\#31101159,\#31201217) and the Research Fund for the Doctoral Program (\#20113418120001). 


\section{REFERENCES}

Cheng YP (2003). The research and application of transgenic maize. Heilongjiang Agr. Sci. 1: 28-31.

Galinat WC (1971). The evolution of sweet corn. Mass. Ag. Exp. Sta. Bull. 591.

Galinat WC (1972). A heritable phenotype for two-ranked ears in maize allelic to the same trait in teosinte. Maize Genet. Cooper. News 46: 108-109.

Giulini A, Wang J and Jackson D (2004). Control of phyllotaxy by the cytokinin-inducible response regulator homologue ABPHYL1. Nature 430: 1031-1034.

Huang H (2003). Recent progresses from studies of leaf development. Chin. Bull. Bot. 20: 416-422.

Kan XZ, Cheng BJ, Chen DS and Zhu MY (2003). The advances in the research on improved variety by ion implantation. Bull. Biol. 38: 11-12.

Li XJ and Meng FJ (1997). Changes of plant hormones during the period of inflorescence differentiation and formation in soybean. Acta Agron. Sin. 23: 446-449.

Li ZT and Zhou Y (1996). The immune determination technology of plant hormones. Nanjing: Sci. Technol. Publishing House of Jiangsu 124-132.

Liu Z, Wang B, Cheng BJ, Hang BH, et al. (1998). Basis studying on using mutual complete sisters recurrent selection on opposite maize. Crop Var. Resources 3: 13-17.

Liu ZY (1982). The genetic analysis of opposite phyllotaxy maize inbred lines. Agr. Sci. Anhui 3: 36-41.

Ma Q, Cheng Y, Qi LL, Xiang Y, et al. (2008). Endogenous hormones content comparison between opposite and alternative maizes. J. Agr. Biotechnol. 16: 995-1000.

Ma Q, Cheng Y, Xiang Y, Zhu SW, et al. (2010). Differentiation of leaf primordium in maize regulated by exogenous auxin. J. Maize Sci. 18: 78-83.

Reinhardt D, Mandel T and Kuhlemeier C (2000). Auxin regulates the initiation and radial position of plant lateral organs. Plant Cell 12: 507-518.

Skoog F and Miller CO (1957). Chemical regulation of growth and organ formation in plant tissues cultured in vitro. Symp. Soc. Exp. Biol. 11: 118-130.

Tan YQ, Xie CX, Jiang HY, Ye H, et al. (2011). Molecular mapping of genes for opposite leafing in maize using simple sequence repeat (SSR) markers. Genet. Mol. Res. 10: 3473-3479.

Tian QY, Cheng B, Wang B, and He MJ et al. (1994). The genetical and isozymatical analyses of the corn's opposite leaf characteristic. Agr. Sci. Anhui 22: 216-218.

Wang B and Gu YL (1995). A kind of new maize germplasm resources-opposite phyllotaxy maize. Crop Var. Resources 4: 31-32.

Wang CX, Sun H, Dong XY and Li P (2008). Studies on the correlation between correlative endogenous hormone contents in leaves and dwarf growth of peach trees. Chin. Agr. Sci. Bull. 24: 226-230.

Xie CX, Zhu SW, Li PJ and Chen B et al. (2002). Obtaining of SCAR markers of two dominant genes for opposite leaves and fruits trait of Zea mays. High Technol. Lett. 12: 38-41.

Zhao LM (2009). Current research advances on plant hormones and regulation of plant development by plant hormones in rice. North Rice 39: 63-69. 